\section{Erfolgt die Übertragung der Infektion mit NTM indirekt?}

Patienten mit zystischer Fibrose (CF) haben ein erhöhtes Risiko sich mit dem multiresistenten nicht-tuberkulösen Mycobacterium (NTM) M. abscessus zu infizieren. Das britische Forscherteam um J. M. Bryant aus Cambridge hat die vollständige Genomsequenzierung (WGS) eingesetzt, um den Akquisitionsmechanismus von M. abscessus bei CF-Patienten zu definieren. Nach ihrer Analyse vermuten die Forscher nun eine indirekte Übertragungsroute. Lancet 2013; 381: 1551-1560

Das NTM M. abscessus verursacht progressive Lungenschäden. Seine Behandlung stellt eine extreme Herausforderung dar. Gegenwärtig ist nicht bekannt, wie die Patienten diesen Organismus erwerben. Die Besorgnis nimmt jedoch zu, dass eine Übertragung von Person zu Person stattfinden könnte. Die britischen Wissenschaftler versuchten mögliche Übertragungsmechanismen in ihrer retrospektiven Kohortenstudie zu ergründen. Dazu haben sie in einem Zentrum für zystische Fibrose zwischen 2007 und 2011 an 168 aufeinanderfolgenden M. abscessus-Isolaten von 31 Patienten vollständige Genomsequenzierungen und antimikrobiel-

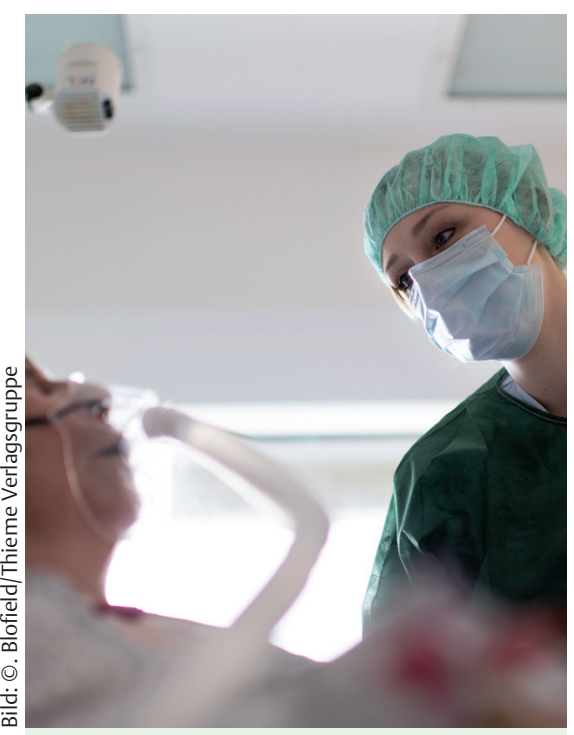

Ob von Mensch zu Mensch oder über Vektoren - der Übertragungsweg von M. abscessus bleibt unklar. le Suszeptibilitätstest untersucht. Parallel dazu unternahmen sie detaillierte Umfeldüberprüfungen auf NTM und definierten potenzielle Übertragungsmöglichkeiten zwischen Patienten sowohl in als auch außerhalb des Krankenhauses, indem sie epidemiologische Daten und Sozialnetzwerkanalysen auswerteten. Im Zentrum für zystische Fibrose galten strenge konventionelle Maßnahmen gegen Kreuzinfektionen, einschließlich strikter Abschottung einzelner Patienten.

\section{Häufige Gelegenheiten zur Ansteckung im Krankenhaus $\nabla$}

Die phylogenetische Analyse deckte bei 11 Patienten 2 gehäufte Ausbrüche mit nahezu identischen Isolaten der M. abscessus-Subspezies massiliense auf, die sich in weniger als 10 Basenpaaren unterschieden. Diese Variation repräsentiert weniger Diversität als die innerhalb von Isolaten von einzelnen Individuen festgestellte Vielfalt, was nachhaltig auf eine zwischenpatientliche Übertragung hinweist. Im Umfeld dieser Ausbrüche hatten alle Patienten zahlreiche Gelegenheiten, sich innerhalb des Hospitals anzustecken. Dagegen scheiterten umfangreiche Stichprobennahmen im Umfeld, die während der Ausbrüche stattfanden, irgendeinen potentiellen punktuellen Ursprung der NTM-Infektion aufzudecken.

Die Cluster der M. abscessus-Subspezies massiliense waren ein Indiz für die Übertragung von Mutationen, die während der Weitergabe der Infektion von einer Person auf andere Patienten aufgetreten waren. So konnten von verschiedenen Personen, die zuvor nie einer Langzeittherapie mit Makroliden oder Aminoglykosiden ausgesetzt waren, Isolate mit konstitutiver Resistenz gegen Ami-kacin und Clarithromycin isoliert werden - ein weiterer Hinweis auf Kreuzinfektionen.

\section{Fazit}

Trotz strenger konventioneller Maßnahmen gegen Kreuzinfektionen im Zentrum für zystische Fibrose deckte die vollständige Genomsequenzierung häufige Übertragungen von multiresistenten NTM zwischen den dort untergebrachten Patienten auf. Obwohl die genaue Übertragungsroute noch nicht feststeht, sind die Ergebnisse dieser epidemiologischen Analyse ein Hinweis auf eine indirekte Übertragung. Die Befunde haben nach Ansicht der Autoren tiefgreifende Auswirkungen auf die Behandlung und Pflege von Patienten mit zystischer Fibrose in Kliniken. Zudem geben sie Anlass, an die Möglichkeit von Kreuzinfektionen in anderen Patientengruppen und mit anderen NTM-Spezies zu denken.

Dr. Volker Kriegeskorte, Buchloe

\section{Kommentar}

B. P. O'Sullivan und C. M. Sassetti vom Howard Hughes Medical Institute in Worcester, MA, USA, halten die Befunde von Bryant et al. hinsichtlich der von ihnen nachgewiesenen Übertragung der M. abscessus-Subspezies massiliense für äußerst wichtig, da die NTM-Infektion als eine immer stärker aufkommende Ursache von Lungenerkrankungen bei Patienten mit zystischer Fibrose gilt. Umso mehr sollten die Forscher effektivere und patientenfreundlichere Therapieschemata gegen NTM-Infektionen entwickeln. In der Zwischenzeit sei es dringend erforderlich, Maßnahmen zur Infektionskontrolle zu verstärken und durchzusetzen, um die Übertragung aller potenziellen Pathogene auf allen möglichen Routen zu verhindern. Lancet 2013; 381: 1517-1519 